\title{
Téoros
}

Revue de recherche en tourisme

\section{La métropole culturelle}

Une nouvelle image de Montréal ?

\section{Martin Drouin}

Volume 25, numéro 2, été 2006

URI : https://id.erudit.org/iderudit/1071070ar

DOI : https://doi.org/10.7202/1071070ar

Aller au sommaire du numéro

Éditeur(s)

Université du Québec à Montréal

ISSN

0712-8657 (imprimé)

1923-2705 (numérique)

Découvrir la revue

Citer cette note

Drouin, M. (2006). La métropole culturelle : une nouvelle image de Montréal ? Téoros, 25(2), 75-77. https://doi.org/10.7202/1071070ar

Ce document est protégé par la loi sur le droit d'auteur. L'utilisation des services d'Érudit (y compris la reproduction) est assujettie à sa politique d'utilisation que vous pouvez consulter en ligne.

https://apropos.erudit.org/fr/usagers/politique-dutilisation/
Cet article est diffusé et préservé par Érudit.

Érudit est un consortium interuniversitaire sans but lucratif composé de l’Université de Montréal, l'Université Laval et l'Université du Québec à Montréal. Il a pour mission la promotion et la valorisation de la recherche. https://www.erudit.org/fr/ 


\section{Chroniques -12}

Chaire de recherche du Canada en patrimoine urbain

\section{La métropole culturelle Une nouvelle image de Montréal?}

\section{Martin Drouin}

Montréal serait appelée à devenir la nouvelle métropole culturelle de l'Amérique du Nord. C'est du moins dans cette perspective que l'entente entre le ministère de la Culture et des Communications du Québec et Tourisme Montréal a permis de doubler le budget de l'organisme qui cherchera désormais à repositionner l'image de la ville sur les marchés nord-américain et européen. "Montréal est une capitale culturelle unique en Amérique du Nord », affirmait d'ailleurs en ce sens Charles Lapointe, président de Tourisme Montréal, dans une entrevue à La Presse (Désiront, 2006 : 6). L'objectif s'inscrit dans une série de stratégies qui façonnera un nouvel avenir pour la ville. Depuis le Sommet de Montréal en 2002, les autorités municipales ont mis sur pied des comités-conseils et attelé les employés de la ville à l'idéation et à la production de documents prospectifs ou réglementaires. En 2004, le plan d'urbanisme était adopté, tandis qu'en 2005 se succédaient la politique du patrimoine, la politique de développement culturelle, le plan stratégique de développement durable, le plan de développement économique de la Communauté métropolitaine montréalaise, la stratégie de développement économique de la Ville de Montréal et les orientations stratégiques de Montréal International. Des documents, tel Montréal, ville de savoir ou Imaginer - Réaliser Montréal 2025. Un monde de créativité et de possibilité, déclinaient à leur tour le « potentiel » de la ville et annonçaient un « plan de match » à suivre pour les prochaines décennies. Les contenus tablaient à l'évidence sur la qualité de vie, l'attractivité et la créativité du lieu. À ce propos, un article publié récemment dans Le Devoir nous apprenait que si Montréal ne pouvait plus aspirer à devenir une métropole financière ou économique, elle avait encore des chances de s'approprier le titre de métropole culturelle ${ }^{1}$ (Harvey, 2006: H3). L'approche promotionnelle de Tourisme Montréal relèverait d'une telle logique en valorisant un avenir culturel comme créneau montréalais.

Tous les intervenants ne s'entendent pas pour dire si Montréal doit jouer dans la cour des grandes villes comme New York, Paris ou Londres ou se positionner plutôt par rapport à des métropoles intermédiaires telles que Barcelone, Dublin ou Stockholm. Quoiqu'il advienne cependant, Montréal aura toujours à se mesurer à sa principale rivale: Toronto. Au-delà de cette portion du débat, les atouts culturels de Montréal seraient des plus réels. II suffit d'écouter ou de lire les propos de Simon Brault, président de Culture Montréal et défenseur passionné du concept de métropole culturelle, pour s'en convaincre $^{2}$ (Brault, 2006 : A7). Dans cette chronique, notons plus

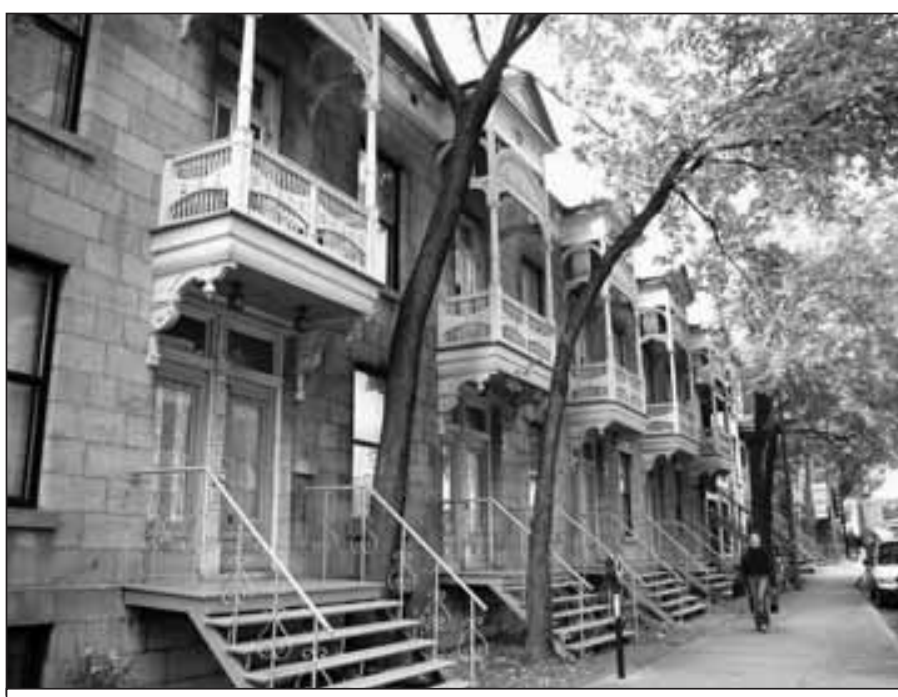

Les maisons de pierre grise du quartier Milton-Parc furent au centre de l'une des campagnes de sauvegarde les plus connues à Montréal.

Photo : Martin Drouin

spécifiquement que la richesse et la diversité du cadre bâti, auxquelles s'ajoute une volonté politique de faire de Montréal une ville de design, s'arriment et bonifient le projet. D'ailleurs, la mise en valeur du patrimoine «bâti, archéologique, paysager et naturel » s'inscrit au premier plan de la création d'un «milieu de vie exceptionnel » et dans l'ambition de créer une « ville ouverte à rayonnement international », deux des cinq axes stratégiques d'intervention du document Imaginer - Réaliser Montréal $2025^{3}$ (Ville de Montréal, 2005 : 9). L'image de la métropole culturelle ainsi valorisée mise sur le visage créatif de la ville, tout en soutenant la revitalisation et la conservation du paysage urbain. La culture et l'expérience urbaine se conjugueraient ainsi pour offrir un produit touristique unique.

Un tel projet pour l'avenir n'avait pas été vu depuis bien des années. Il faut probablement remonter à la « métropole du progrès » de Jean Drapeau dans les années 1960 pour retrouver un même entrain prospectif. À l'époque où Toronto s'imposait déjà comme la véritable métropole économique du Canada, l'ancien maire se prenait à croire qu'il pourrait transformer Montréal et l'inclure dans le club sélect des grandes villes internationales. À l'instar des cités qui avaient marqué l'histoire de l'humanité, comme Athènes, Rome, Paris ou Londres, Montréal était alors destinée à devenir la première ville mondiale du XXIe siècle. Le projet s'inscrivait dans l'air du temps, alors que de 
nombreuses villes nord-américaines se métamorphosaient sous l'influence de l'idée de progrès et d'un futur pensé sous un jour nouveau. Une politique de grands projets, favorisée par une santé économique, d'abord vive puis de plus en plus chancelante, marqua Montréal pendant deux décennies ; il est d'ailleurs symptomatique que le document Imaginer - Réaliser 2025 inscrive dans sa signature graphique des représentations de cette période, à savoir I'Exposition universelle de 1967 et les Jeux olympiques de 1976. La création d'une " métropole du progrès » fut progressivement abandonnée à la fin des années 1970. Les rêves de grandeur s'évanouirent à mesure que les autorités municipales durent s'ajuster aux nouvelles réalités de l'époque. Finalement, elles se résignèrent à annoncer la « renaissance de la ville traditionnelle» (Turcotte, 1976).

L'image de Montréal aujourd'hui valorisée ne date pas des derniers plans d'avenir des élus municipaux. Elle s'est paradoxalement construite dans la contestation de l'idée de la « métropole du progrès ". Malgré l'élan qui permit à la ville de s'ajuster aux conditions de vie urbaine du XXe siècle et de se démarquer, graduellement, les Montréalais ne se reconnurent plus dans le projet. La ville semblait offerte aux étrangers. Le thème de la dépossession était en effet récurrent dans la revendication d'une ville autre que celle qui brillait alors sur la scène internationale. Différents contentieux étaient ainsi formulés, avec en tête de liste la sauvegarde du patrimoine urbain. II fallut d'abord déboulonner les conceptions classiques du monument historique pour faire accepter la valeur d'éléments hétéroclites et peutêtre moins flamboyants. La ville ne possédait pas, comme à Paris, un ensemble d'immeubles haussmaniens qui unifiait son image, mais les « plex » de brique rouge et de pierre grise, sertis d'escaliers et de corniches, n'en nécessitaient pas moins d'être conservés. Des quartiers animés et vivants - ceux que l'on désigne aujourd'hui de créatifs - existaient encore à proximité du centre-ville moderne. Peu à peu, un cadre de vie signifiant, pour ceux qui l'investissaient et en réclamaient la protection, donnait à Montréal un « je-ne-sais-quoi », comme on aurait dit à une autre époque, pour qualifier la ville. En marge d'une modernité nord-américaine rutilante, les Montréalais inventaient un Montréal de l'ordinaire, à l'enseigne d'une urbanité dé-

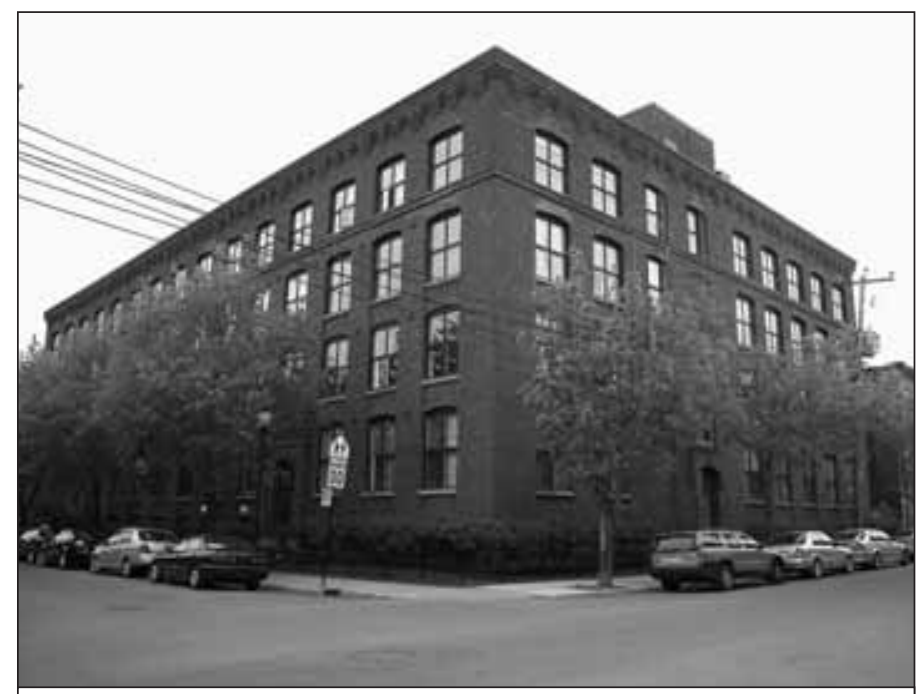

Une ancienne manufacture, rue Demers dans le quartier Plateau-Mont-Royal, est aujourd'hui convertie en immeuble en copropriété.

Photo: Martin Drouin linquante et rebelle, ancré dans un environnement patrimonial qui dépassait la somme des monuments distincts, dans lequel le quotidien des habitants eux-mêmes le transformait en un cadre de vie. Bref, une ville que Ricardo Florida, gourou des villes créatives et inventeur de l'indice bohémien, n'hésitait pas à qualifier récemment de «l'un des secrets les mieux gardés d'Amérique du Nord » et «l'une des 'étoiles montantes' des économies créatives » (Stolarick, 2005 : 2).

De la même manière dont la « métropole du progrès » était inscrite à l'époque dans l'air du temps, la métropole culturelle et créative semble l'être tout autant. Au début du mois d'avril, Le Devoir nous apprenait que New York, Toronto, Philadelphie et Los Angeles convoitaient le même titre. La première décidait de créer un bureau municipal de développement culturel pour défendre son titre de capitale des arts et de la culture (Soucy, 2006 : B6). L'annonce faisait suite au dévoilement du rapport intitulé « Creative New York », produit par le Center for an Urban Future, dont l'une des consultantes était appelée à Montréal (Pélouas, 2006 : 16). La seconde, semblablement intéressée par le titre, investissait massivement dans des infrastructures culturelles et faisait appel aux services de Richard Florida (Robitaille, 2005 : A6), le même qui avait précédemment travaillé à la revitalisation de Lower Manhattan ${ }^{4}$ (Florida, 2002a) et que les autorités montréalaises invitèrent au début de l'année 2005. Pour saisir l'ampleur du phénomène, il suffit d'ailleurs de consulter le site Internet de la compagnie qu'il a créée pour répondre à la demande, à la suite de la publication de l'ouvrage The Rise of Creative Class (Florida, 2002b) $^{5}$. Dans un autre registre outre-atlantique, le programme des capitales européennes de la culture, adopté en 1999 par l'Union européenne, démontre l'importance accordée à l'alliance entre ville, culture et rayonnement : le succès de Lille en 2004 le prouve $^{6}$. II est flatteur de croire que Montréal pourrait avoir l'étoffe d'une métropole culturelle. Toutefois, à constater l'intérêt non dissimulé qu'ont les institutions à vouloir s'imposer dans le club sélect des villes culturelles et la rude concurrence qui en découle, le projet devrait inclure, à l'évidence, les Montréalais qui avaient jadis contesté la construction d'une ville qui ne leur appartenait plus et favorisé l'émergence d'un autre Montréal, dont on veut vendre l'image aujourd'hui. En ce sens, le maire Gérald Tremblay disait juste lorsqu'il affirmait: "Ce sont les Montréalais et les Montréalaises qui font Montréal, qui lui donnent sa personnalité si unique, son âme, sa force » (Tremblay, 2006).

Certains regrettent que le projet de Montréal, métropole culturelle, n'accorde pas une plus grande place aux gestes architecturaux d'envergure. L'éternelle rivale est alors posée en exemple. En effet, Toronto verra, entre autres, l'agrandissement du Royal Ontario Museum, réalisé par l'architecte Daniel Libeskind, qui travaille aussi à l'édification des Freedom Towers à New York, et la construction du Musée des beaux-arts de l'Ontario, par l'architecte Frank O. Gerhy, I'homme qui conçut le musée de Bilbao à l'origine de la renaissance de la ville basque. Depuis quelques années pourtant, des gestes architecturaux sont venus marquer Montréal. On peut penser à la Grande Bibliothèque, au Palais des congrès ou aux nouveaux pavillons des universités montréalaises. II est intéressant de noter que les bâtiments furent presque toujours jugés en opposition avec le milieu patrimonial environnant pour en évaluer la valeur. On n'a qu'à se rappeler le sobriquet donné à la façade de la rue Sherbrooke du complexe Pierre-Dansereau. Mis à part la réputation internationale des concepteurs, il n'est pas certain qu'un bâtiment de Libeskind ou de 


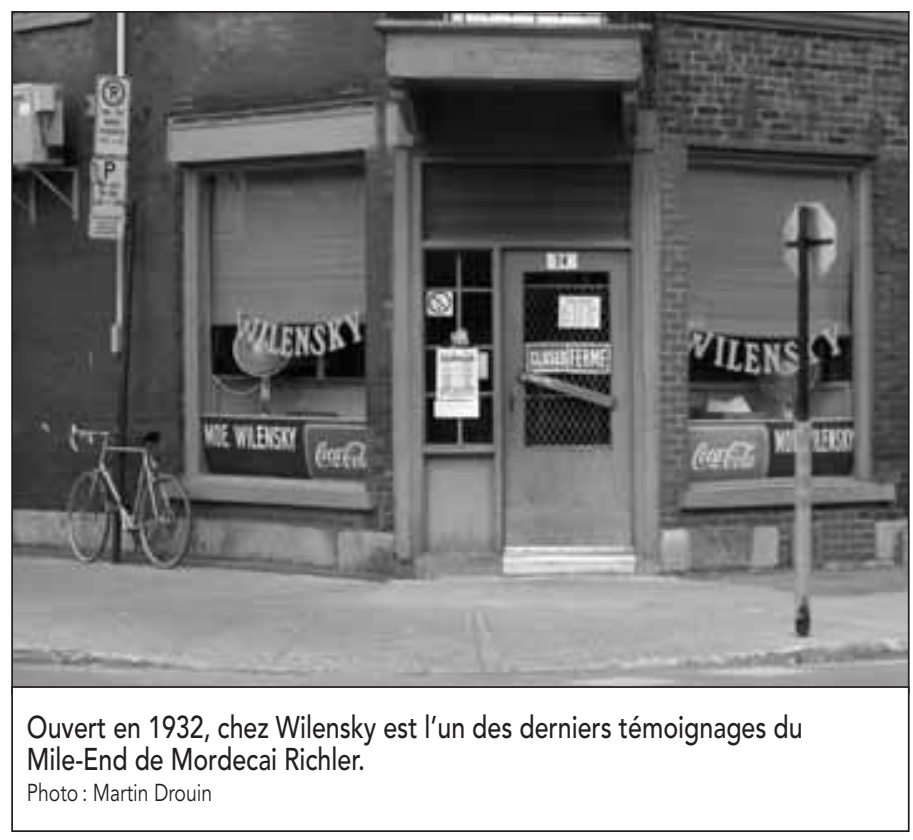

Gerhy en plein cœur du centre-ville fasse l'unanimité ${ }^{7}$. À titre d'exemple, le dernier geste de la " métropole du progrès ", le Stade olympique, même s'il s'affirme comme un lieu quasi incontournable dans le circuit des visiteurs étrangers et l'un des édifices dont l'image est la plus reproduite, souffre encore d'un manque de reconnaissance dans la ville. Des monuments phares d'une métropole culturelle seraient-ils semblablement reçus ? À cela, on peut répondre que Montréal n'est pas Toronto.

Le « bric-à-brac » montréalais, pour reprendre l'expression des éditeurs de l'ouvrage Montréal, L'oasis du Nord, peut-il résister au projet de métropole culturelle (Boivin et Comeau, 1992 : 11) ? De nombreuses questions émergent. L'identification visuelle des quartiers, par exemple, pourrait bien sûr faciliter la qualification de la ville, mais au risque cependant d'un amenuisement de sens et d'une idéalisation de la vie urbaine montréalaise. II existe bel et bien un écart entre l'idée du Plateau de Michel Tremblay, du Mile-End de Mordecai Richler, de la Petite Patrie de Jean-Claude Germain, du Saint-Henri de Gabrielle Roy et le quotidien des habitants de ces quartiers. On pourrait rétorquer qu'il faudrait procéder à des zonages de protection culturelle pour contenir l'escale foncière et l'embourgeoisement des quartiers. De la même manière qu'il fallut œuvrer contre la tentation de «muséification » du Vieux-Montréal alors que sa valeur historique était révélée, il faudrait s'interroger sur la « culturalisation » des quartiers centraux alors que leur valeur a été identifiée. En définitive, bien des paradoxes peuvent apparaitre dans une telle entreprise de mise en valeur et de médiation. Ouvrir Montréal au tourisme international par le biais de ses atouts culturels peut être une chance unique de valoriser ce que la métropole peut offrir, à condition toutefois de ne pas pourrir les fruits de la récolte. Un autre Montréal pourrait rapidement émerger dans une forme que l'on ne soupçonne pas. Rien n'est éternel dans l'univers des représentations.

Martin Drouin est historien et docteur en études urbaines. II est coordonnateur de l'Institut du patrimoine à l'UQAM.

\section{Notes}

1 Le journaliste rapportait les propos de Benoit Labonté, maire de l'arrondissement Ville-Marie et responsable de la culture au comité exécutif de la Ville de Montréal.

2 Lire des extraits de la communication prononcée dans le cadre du colloque Réinventer Montréal, organisé par le Département d'études urbaines et touristiques de l'Université du Québec à Montréal pour souligner son trentième anniversaire, à la page « Idées » du Devoir.

3 Les trois autres axes sont la "ville de savoir, de création et d'innovation ", la « métropole culturelle d'avant-garde » et la « ville d'infrastructures performantes".

4 On peut consulter le rapport sur le site Internet de la compagnie créée par Ricardo Florida à l'adresse suivante: [www.catalytix.biz/acrobat/ NYRPA.pdf].

5 Richard Florida Creativity Group (2006), [www.creativeclass.org], (consulté le 5 mai 2006).

6 Europa, le portail de l'Union européenne (2006), « Capitales européennes ", [http://europa.eu.int/comm/culture/eac/other_actions/cap_ europ/cap_eu_fr.html], (consulté le 5 mai 2006).

7 On peut visualiser le travail de Libeskind et de Gerhy sur les sites Internet des deux institutions (cf. Musée des beaux-arts de l'Ontario (2006) et Royal Ontario Museum (2006).

\section{Bibliographie}

Boivin, Robert, et Robert Comeau (dir.) (1992), Montréal, L'oasis du Nord, Paris, Autrement.

Brault, Simon (2006), «Montréal, une métropole culturelle inachevée », Le Devoir, 27 avril, p. A7.

Désiront, André (2006), « Montréal misera plus sur le volet culturel », La Presse, 22 mars, cahier «L'Actuel », p. 6.

Florida, Ricardo (2002a), Rebuilding Lower Manhattan for the Creative Age: Implications for the Regional Plan Association and Civic Alliance, rapport préparé pour la Regional Plan Association and Civic Alliance, 20 p.

Florida, Richard (2002b), The Rise of Creative Class: And How It's Transforming Work, Leisure, Community and Every Day Life, New York, Basic Book, 430 p.

Harvey, Réginal (2006), « La mise au point de toutes les énergies sur le plan culturel », Le Devoir, 29 avril, p. H3.

Musée des beaux-arts de l'Ontario (2006), Site Internet officiel, [www.ago.net], (consulté le 5 mai 2006).

Pélouas, Anne (2006), « Montréal-New York, même combat ! », Voir, vol. 20, $n^{\circ} 12,23$ mars, p. 16.

Robitaille, Antoine (2005), "Florida, le gourou de Toronto », Le Devoir, 3 septembre, p. A6.

Royal Ontario Museum (2006), Site Internet officiel, [www.rom.on.ca], (consulté le 5 mai 2006).

Soucy, Louise-Maude (2006), « New York sort les griffes : La ville défend son titre de capitale des arts et de la culture ", Le Devoir, 6 avril, p. B7.

Stolarick, Kevin, Richard Florida, et Louis Musante (2005), Montréal, ville de convergences créatives : Perspectives et possibilités, Document de synthèse du groupe Catalytix, [www.culturemontreal.ca/pdf/050127_cata lytix_fr.pdf], (consulté le 2 mai 2006).

Tremblay, Gérald (2006), Imaginer - Réaliser Montréal 2025. Un monde de créativité et de possibilité, Allocution inaugurale du Colloque Réinventer Montréal, lors du $30^{\circ}$ anniversaire du Département d'études urbaines et touristiques, UQAM, [www.ville.montreal.qc.ca/montreal2025], (consulté le 2 mai 2006).

Turcotte, Claude (1976), "L'hôtel de ville s'occupe enfin d'affaire municipale, La Presse, 7 février.

Ville de Montréal (2005), Imaginer - Réaliser Montréal 2025. Un monde de créativité et de possibilité, Montréal, Service de la mise en valeur du territoire et du patrimoine, Direction de la planification stratégique. 